\title{
Aeronautical Emergency and Abnormal Checklists: Expectations and Realities
}

\author{
Barbara K. Burian, Ph.D. \\ San Jose State University at NASA Ames Research Center
}

\begin{abstract}
Developers of emergency, abnormal, and non-normal checklists hold a number of beliefs about how, when, and under what types of conditions flight crews will access and use these checklists. These beliefs or expectations strongly influence the decisions developers make about checklist content, design, and presentation. Interviews with pilots involved in incidents and accidents, simulator observations, and analyses of paper and electronic checklists, reveal that many of the expectations developers hold, which are implicit in checklist designs, do not match the realities of emergency and abnormal situations and flight crew checklist use. Several of these expectations are presented along with contrasting realities and some suggested design solutions.
\end{abstract}

\section{Introduction}

Emergency, abnormal, and non-normal checklists are essential tools that flight crews use to structure and guide their responses to abnormal conditions and emergency situations aboard aircraft. These checklists are typically comprised of at least one, but often all, of the following: a list of actions to be performed, important information that has bearing on the accomplishment of actions or the on-going operation of the aircraft, and decision points that guide the crew to specific checklist sections based on the exact circumstances with which they are faced.

In civil aviation, emergency, abnormal, and nonnormal checklists are largely text based and are presented to crews in paper or electronic formats. The developers of these checklists have certain expectations about how, when, and under what conditions they will be used by flight crews during emergency or abnormal situations. These expectations powerfully influence developers' design of checklists but are not always explicit or correct - it appears that at least in some cases, designers have made implicit assumptions without examining the basis for those assumptions. It is essential that the expectations that guide checklist design match the realities of emergency and abnormal situations and checklist use by the flight crews if they are to be effective tools.

\section{Practice Innovation}

A thorough examination and analysis of emergency, abnormal, and non-normal checklists used in aviation was undertaken as a part of the Emergency and Abnormal Situations Study at the NASA Ames Research Center. Many aspects of checklist design, development, presentation format, and use were of interest. Analyses have led to what some may consider to be a shocking conclusion: there is no such thing as "a perfect checklist." This means that not only are there limitations in all current checklists but also that there will likely always be limitations in emergency and abnormal checklists. For example, it is improbable that a single checklist could be designed so that it perfectly addresses all possible conditions under which it might ever be needed.
Having said this, however, it is clear that current checklist designs can be improved substantially. There may be no "perfect checklist" but checklists should be easy to use, they should accommodate human performance limitations under stress, and their design should be consistent with the realities faced by the crews using them. In this study over 50 crew members involved in emergencies, abnormal situations and accidents were interviewed and a similar numbers of pilots were observed during simulator training. Analyses of incident reports filed by pilots with the Aviation Safety Reporting System (ASRS) and aircraft accident reports from around the world were conducted, and a structured coding form was used to analyze electronic and paper checklists from over 30 major aircraft manufacturers and part 121 air carriers. Through these interviews, observations, and analyses, several expectations underlying many aspects of checklist design that are at odds with the realities of emergency and abnormal checklist use by flight crews were identified.

\section{Findings and Discussion}

Some of the most commonly identified expectations that are implicit in the design of many current emergency, abnormal, and non-normal checklists are presented below along with brief descriptions of contrasting realities.

Expectation: Crews will correctly interpret the cues available to them and will know which checklist to accomplish for their situation.

Reality: Unfortunately, it is not uncommon for crews to misconstrue abnormal condition cues. For example, an alert message indicating low oil pressure can easily lead crews into accomplishing a Low Oil Pressure checklist when in fact, an engine has ceased to operate, causing the oil pressure to be low, and the Loss of an Engine checklist is the proper one to complete. This error has often been observed during simulator training sessions. Similarly, instructors at several air carriers have reported that crews commonly misinterpret flight control problem cues and, for example, select a checklist for problems with moving leading edge devices when the correct checklist to accomplish pertains to asymmetrical 
leading edge devices (Berman, Burian, Dismukes, \& Geven, 2006).

Additional information that would assist crews in making fine distinctions between similar situations that require different responses, such as with flight control malfunctions, is rarely provided in checklists. Checklists could help avoid such confusions by directing crews to look for additional cues to ensure that they have not mistaken their situation for another. One major aircraft manufacturer is in the process of revising all of their non-normal checklists and such information will be provided in some of these newly revised checklists (Holder \& McKenzie, 2006).

Expectation: A checklist will exist for the situation encountered.

Reality: In March of 2000, two alerts were displayed in the cockpit of a B737-700 as it climbed through 1000 feet after takeoff. The alerts indicated that the airspeed and altitude displayed on the captain's panel did not agree with those values displayed on the first officer's panel. The climb and cruise pages on the flight management computer display lacked any data output and the progress page showed no distances or times between route segments. As the situation unfolded a variety of other anomalies were noted: the captain's altimeter indicated a climb but lagged behind the first officer's altimeter, the wind readout arrows on the captain's and first officer's displays differed by almost $180^{\circ}$, and the captain's altimeter and airspeed indicators finally disappeared from the cockpit displays all together. The captain consulted emergency and abnormal checklists but could find no checklist that pertained to their situation. Fortunately, this flight occurred during good weather conditions and was able to return to the airport from which they had just departed. Upon landing, maintenance personnel discovered that a vane that protrudes from the outside of the aircraft, which provides airspeed and altitude information to the computers for the captain's cockpit displays, was badly damaged.

Interviews with many pilots and reviews of reports filed with the Aviation Safety Reporting System have similarly revealed events for which there were no checklists. Michel Tremaud (2002) studied a number of incidents reported to a major aircraft manufacturer and found that quite often the emergency or abnormal situations encountered by the flight crews in these incidents went "beyond the scope of the published procedures.” In some cases no procedure or checklist existed for the situation but in others, the checklist actions and guidance given did not address the specific situation faced by the crews.

In truth, it is not possible for checklist designers to anticipate every conceivable failure or malfunction that might occur on an aircraft. However, checklists should be developed for those that are fairly common or could be reasonably predicted (Burian, Barshi, \& Dismukes, 2005). Additionally, modern aircraft displays and controls rely heavily upon computers; checklist designers must be diligent in thinking through all the possible failure modes that might occur when erroneous information is sent to aircraft computers and develop checklists accordingly. I have also identified a number of other checklist design factors, such as considerations of terrain or adverse weather conditions, that checklist developers should consider to minimize the number of times that crews find the checklists they are to use don't quite account for some of the specifics of their situations (Burian 2006).

Expectation: Crews will remember the correct title of the checklist they wish to perform and will know how to locate it.

Reality: Flight crews often do not recall the titles given to a specific checklist, which causes a delay in identifying it in a table of contents, index, or electronic checklist menu. Additionally, checklists are not always located where flight crews can find them easily. In 1980, the crew of Saudi Arabian Airlines flight 163 unsuccessfully searched for a cargo fire checklist for several minutes in the "Abnormal” section of their Quick Reference Handbook (QRH - a manual comprised of checklists). The investigation into this accident revealed that the checklist the crew was looking for but never found was filed in the "Emergency" section of the QRH instead, and all 301 people on board perished (Flight Safety Focus, 1985).

Checklist developers must remember that the flight crews who use their checklists will most likely never be as familiar with them as the developers are. Multiple methods and approaches for checklist identification and access should be incorporated in an electronic checklist system or QRH to accommodate the variety of ways in which a pilot may try to find a particular checklist. For example, in a QRH index, the checklist for air conditioning smoke could be listed under "A" as "Air Conditioning Smoke" and under "S" as "Smoke - Air Conditioning” regardless of the actual checklist title.

Similarly, given the importance of locating this checklist quickly, the checklist itself might be included in two different sections within the QRH: in the air conditioning and pressurization section as well as in the smoke and fire section. Thus, if crews turn directly to one of these sections without first consulting the index, they will still find the checklist.

Advanced caution and warning systems, such as Boeing's Engine Indicating and Crew Alerting System (EICAS) and Airbus's Electronic Centralized Aircraft Monitoring system (ECAM), can alert the crew to a wide variety of emergency and abnormal conditions. Checklist developers typically give the checklists for those conditions the same names as their associated alerts, thus facilitating the identification of the correct checklists. Furthermore, in those aircraft equipped with integrated electronic checklists, the crew alerting system can even cause the checklists that correspond to displayed alerts to be automatically presented for crew completion. (Integrated electronic checklists are those that are linked to aircraft systems and controls through a network of sensors.) Obviously, this is a distinct advantage of integrated electronic checklists over those printed on paper (Boorman, 2000).

Expectation: Crews will access and use available emergency and abnormal checklists.

Reality: Most often, when crews are faced with an emergency or abnormal situation, they will try to locate and 
complete the checklists appropriate for their condition. That is what these checklists are for and a good portion of flight crew initial and recurrent training is devoted to responding to emergency and abnormal situations and familiarization with the appropriate checklists. However, there are times that even when the proper checklists exist for a given situation, it may not be practicable for crews to access and complete them.

In 1988, as a B737-200 was leveling off at 24,000 ft., an 18-foot section of fuselage separated from the aircraft. (National Transportation Safety Board (NTSB), 1989). In the 13 minutes that it took the flight crew to perform an emergency descent and landing, they completed all or significant parts of 17 different checklists-largely from memory. During the descent, the crew only had time to consult the emergency and abnormal checklists once, to find the reference speed for a landing with reduced flaps (M. Tompkins, personal communication, April 25, 2003).

Similarly, the pilot who filed the following report with the Aviation Safety Reporting System (ASRS) also described an increase in workload in response to an emergency situation that precluded accessing emergency checklists - in this case, dealing with an electrical failure during approach and landing: “The...events took place over a time span of less than 4 minutes during a critical phase of flight...the events occurred simultaneously with radio transmissions, configuration changes, airspeed changes and constantly changing altitude... What we learned from this event is that running the emergency checklists may not be a classical situation where one has plenty of time for analysis and application of curative measures” (Accession \#437830; Aviation Safety Reporting System, 2001).

Thus, some situations unfold so quickly or are so time-critical that all the crews' attention must be devoted to controlling and landing the airplane with little or no time to spare for consulting an emergency checklist. There may also be rare occasions in which the emergency situation itself renders available checklists inaccessible to the crew. For example, in 1989, the flight crew of United 811 experienced an explosive decompression over the ocean at approximately 22,500 thousand feet after departing Honolulu, Hawaii (NTSB, 1992). The force of the decompression caused the door to the cockpit to separate and the flight engineer's emergency and abnormal checklists were sucked out of the cockpit and ended up spread all over the floor of the B747's first class compartment.

There is little that checklist designers can do to accommodate crews whose workload is so high that they are unable to access emergency and abnormal checklists or crews whose once accessible checklists become inaccessible. These are scenarios that should be addressed during training. However, as is discussed next, the amount of time available for dealing with emergency or abnormal situations is an important factor for checklist designer consideration.

Expectation: Adequate time will be available to complete all actions included in a checklist.

Reality: The paper checklist developed by one manufacturer to be used in the event of an electrical failure on a particular type of aircraft is 12 pages long (Burian, 2005).
Also, the Transportation Safety Board (TSB) of Canada estimated that the in-flight smoke and fire checklists used by the flight crew of Swissair 111 in 1998, could have taken as much as 30 minutes or more to accomplish. The aircraft crashed into the ocean approximately 20 minutes after the crew first detected an unusual odor on the flight deck (TSB of Canada, 2003).

Clearly, some checklists are quite long either in physical length (i.e., number of items to be performed), in duration (i.e., amount of time it takes to complete the actions stipulated), or both. If it is too late to alter the design of an aircraft system to eliminate the need for lengthy procedures, checklist designers can insert alternate actions at various points throughout a lengthy checklist, which can be selected by pilots when time is running short. For example, in several locations within a smoke and fire checklist crews might be directed to abandon the checklist and focus on landing the aircraft if the situation is uncontrollable or when landing is imminent (Flight Safety Foundation, 2005).

Expectation: Emergency or abnormal checklist actions will be successful in resolving the problem or at least in stabilizing the situation.

Reality: It is not unusual for crews to complete all the actions on a checklist and still find that their situation has not been resolved or stabilized. For example, in 2000, the crew of Alaska Airlines flight 261 completed all available relevant checklists but continued to have difficulty with their horizontal stabilizer trim (NTSB, 2002).

Only a few emergency and abnormal checklists include any type of guidance about what crews should do in the event that checklist actions are ineffective and usually this guidance takes the form of an instruction to land at the nearest suitable airport. Information about what crews should and should not do when a checklist "fails" should be included in checklists for situations that have critical implications for continued safe flight.

Expectation: Crews' cognitive capabilities will be unimpaired during emergency and abnormal conditions and they will be able to perform complex mental calculations without difficulty.

Reality: Au (2005) studied the ability of pilots to accurately recall and perform items from emergency and abnormal checklists that must be accomplished without reference to a printed checklist (i.e., memory items). He found that pilots committed numerous errors in recalling the memory items correctly even during conditions that were not stressful. Similarly, during simulator training sessions several crews were observed having difficulty applying multipliers to landing distances as required when experiencing various system failures. A QRH table indicated that normal landing distances were to be multiplied by decimals ranging from 1.1 to 1.725 to determine the new (and longer) distance needed to land with an inoperative system. On occasion, simulator instructors suggested that crews "just double the landing distance required and not get bogged down in multiplication.” Cognitive performance limitations under high workload and stress are often not considered when developers design emergency and abnormal checklists. Tunneling and 
fixation of attention, restrictions in working memory, difficulty in shifting mental sets, and other cognitive processing difficulties can commonly occur with the high stress and workload of emergency and abnormal situations. Much can be done with the design of checklists to accommodate these limitations. For example, a few air carriers have eliminated the need for memory items by printing those steps on a card that can be readily accessed by flight crews. Once these steps have been completed by referencing the card, any remaining checklist items are then located in the QRH for completion.

Expectation: Pilots will not troubleshoot or "go beyond" the checklist.

Reality: As mentioned earlier, the crew of Alaska Airlines flight 261 experienced considerable difficulty with their horizontal stabilizer trim. After completing the relevant checklist actions, they continued to troubleshoot the problem, in part to determine controllability of the aircraft and also, apparently, to see if they could resolve the situation which previously completed checklist actions did not rectify. This troubleshooting appears in fact to have exacerbated their difficulties in controlling the aircraft, and they eventually crashed into the Pacific Ocean (NTSB, 2002).

Similarly, during simulator training sessions flight crews have quite often been observed performing a variety of actions to "troubleshoot" their situation before accessing emergency and abnormal checklists. Some of these actions have allowed the crews to ascertain what their exact situation was, so they could determine the most appropriate checklist to complete. However, in other circumstances, the crews were often just trying to complete non-memory and non-time critical actions from memory without accessing the checklist.

Completing non-memory items without reference to a checklist has also resulted in serious incidents in flight, not just during training sessions. In 1996, the flight engineer aboard a B727 responded to an airconditioning PACK that had "tripped off" without consulting the checklist. Instead of closing the outflow valve, as instructed by the checklist, the flight engineer actually opened it and the aircraft rapidly lost pressurization. During this event, the captain, flight engineer, and lead flight attendant, who had been on the flight deck at the time, each briefly lost consciousness. The first officer, who was the pilot flying, donned his oxygen mask immediately and performed an emergency descent and landing (NTSB, 1998a).

There is really not anything that checklist designers can do about crews who complete actions without referencing a checklist. They can, however, include caution statements in checklists that warn crews about further actions they should not attempt in certain situations, such as continued troubleshooting of flight control problems.

Expectation: All instructions in the checklist will be clear to the pilots and they will know the proper way to complete all of the actions mentioned.

Reality: Pilots are often confused by checklists and subsequently make errors when completing checklist actions. To illustrate, one co-pilot filed the following report to the Aviation Safety Reporting System: "I called for the QRH for the loss of hydraulic pressure. While the Captain read the QRH procedure, he was having some difficulty identifying the exact nature of the failure as well as the proper corrective action...I feel that more time should be spent on QRH familiarization during training. The QRH [for this type of aircraft] is a bit confusing in places and actually contains mistakes” (Accession \#440922; ASRS, 2001). Similarly, in 1996, the flight engineer on FedEx 1406 appeared to have been confused by one of the actions stipulated on a checklist for in-flight fire and did not accomplish two other steps on that checklist (NTSB, 1998b). These errors resulted in the aircraft still being partially pressurized at landing and delayed the crew's emergency evacuation.

It can be difficult for checklist designers, who are intimately acquainted with every checklist item they have written, to identify aspects that might confuse flight crews. During development and checklist validation, it is important to have several line pilots (as opposed to test pilots) with varying amounts of experience, who are uninvolved in checklist development, evaluate checklists in quiet rooms (i.e., table-top evaluations), and use them in simulators under the most realistic flight conditions possible. Burian (2006) also includes other things that checklist developers can do to make emergency and abnormal checklists as clear and easy to use as possible.

\section{Conclusion}

Designing an emergency or abnormal checklist that is clear, complete, easy to execute, and that supports flexible crew response to multiple scenarios is very difficult, and little guidance exists for checklist developers about how to design the best product possible. This gap was being addressed by work conducted under Emergency and Abnormal Situations Study that had been funded under NASA's Aviation Safety and Security Program. A comprehensive model of all aspects of emergency and abnormal checklist design and content was developed (Burian, 2006). This model addresses human factors issues of emergency and abnormal checklist design and presentation, human performance considerations under stress, situational and operational demands, and the degree to which checklists appropriately guide and structure situation management and response. It is essential that comprehensive guidance and best practices in emergency and abnormal checklist design be determined and provided to checklist designers to support the development of the most "perfect" checklists possible.

\section{Acknowledgements}

I extend my sincere thanks to the numerous pilots, instructors, check airmen, engineers, and checklist developers who have generously shared their experiences, insight, wisdom, and concerns to further this work. The admiration I have for their professionalism and dedication is boundless. I would also like to acknowledge members of the Emergency and Abnormal Situations Study team, particularly Ben Berman, Richard Geven, Sean Belcher, and Captain Richard 
Fariello who have contributed greatly in identifying issues in the design of emergency and abnormal checklists. Last, but certainly not least, I would also like to thank Captain David Keeling, Key Dismukes, and three anonymous reviewers who provided very helpful comments and suggestions regarding earlier drafts of this paper.

\section{References}

$\mathrm{Au}, \mathrm{H}$. (2005). Line pilot performance of memory items. In Proceedings of the $13^{\text {th }}$ International Symposium on Aviation Psychology, Oklahoma, City, OK.

Aviation Safety Reporting System. (2001). Search Request Number 6133. NASA Ames Research Center, Moffett Field, CA.

Berman, B., Burian, B, K., Dismukes, R. K., \& Geven (2006). Current practices in training Part 121 flight crews for emergency and abnormal situations. Manuscript in preparation.

Boorman, D. (2000). Reducing Flight Crew Errors and Minimizing New Error Modes with Electronic Checklists. In the Proceedings of the International Conference on Human-Computer Interaction in Aeronautics, 2000. Polytechnic International Press, Toulouse, France.

Burian, B. K. (2006). Emergency and abnormal checklists: Design, content, and use. Manuscript in preparation.

Flight Safety Focus. (1985, May). In-flight fire: Report of the Saudi Arabian Civil Aviation Authority. Flight Safety Journal,1-12.

Flight Safety Foundation. (2005). Flight crew procedures streamlined for smoke/fire/fumes. Flight Safety Digest, 24 (6), 31-36.

Holder, B. \& McKenzie, W. (2006). Quick Reference Handbook: Summary of changes. Presentation given at the Boeing 2006 Flight Operations Symposium, Seattle, Washington. May 2-3.
National Transportation Safety Board. (1989). Aloha Airlines Flight 243, Boeing 737-200, N73711, Near Maui, Hawaii, April 28, 1988. Washington, DC: NTSB (NTIS No. PB89-910404).

National Transportation Safety Board (1992). Aircraft Accident Report - Explosive Decompression-Loss of Cargo Door in Flight, United Airlines Flight 811, Boeing 747-122, N4713U, Honolulu, Hawaii, February 24, 1989. Report Number NTSB AAR92/02. Washington, DC: NTSB

National Transportation Safety Board. (1998a). American Trans Air Flight 406, Boeing 727-290, N775AT, Indianapolis, Indiana, May 12, 1996. (NTSB ID No. CHI96IA157).

National Transportation Safety Board (1998b). Aircraft Accident Report - In-flight Fire/Emergency Landing, Federal Express Flight 1406, Douglas DC-10-10, N68055, Newburgh, New York, September 5, 1996. Report Number NTSB AAR-98/03. Washington, DC: NTSB.

National Transportation Safety Board. (2002). Loss of Control and Impact with Pacific Ocean, Alaska Airlines Flight 261, McDonnell Douglas MD-83, N963AS, About 2.7 Miles North of Anacapa Island, California, January 31, 2000. Washington, DC: NTSB. (NTIS No. PB-2002-910401).

Transportation Safety Board of Canada (2003). Aviation Investigation Report A98H0003, In-flight Fire Leading To Collision with Water, Swissair Transport Limited McDonnell Douglas MD-11 HB-IWF, Peggy's Cove, Nova Scotia 5nm SW, 2 September 1998. Gatineau, Quebec, Canada: TSB of Canada.

Tremaud, M. (2002). Operational and Human Factors Involved in Situations Beyond the Scope of Published Procedures. Presentation given at the Airbus $16^{\text {th }}$ Human Factors Symposium, Singapore, October 810. 